\title{
Growth of $\mathrm{Hg}_{1-x} \mathrm{Cd}_{x} \mathrm{Te}$ single crystals by travelling heater method under accelerated crucible rotation conditions
}

\author{
R.U. Bloedner and P. Gille \\ Department of Physics, Humboldt Unicersity of Berlin, Invalidenstrasse 110, D-O-1040 Berlin, Germany
}

Received 19 June 1992; manuscript received in final form 23 November 1992

The accelerated crucible rotation technique (ACRT) has been applied to the THM growth of $\mathrm{Hg}_{1-x} \mathrm{Cd}_{x} \mathrm{Te}$ crystals to grow the crystals at a higher rate. These higher growth rates, which should be achieved by extending the regions of convectional stirring towards the interfaces, have been used in an attempt to explain the results in terms of simple constitutional supercooling arguments. Some different ACRT cycles which fulfil simple hydrodynamic and geometric criteria have been studied. The grown crystals were investigated with respect to their metallurgical homogeneity and their structural perfection. These properties have not been degraded by increasing the growth rate from 1.5 to $8.5 \mathrm{~mm}$ per day.

\section{Introduction}

The growth of $\mathrm{Hg}_{1-x} \mathrm{Cd}_{x} \mathrm{Te}$ single crystals by the travelling heater method (THM) is beneficial for two reasons. Firstly, the segregation effects are minimized by steady-state conditions and a homogeneous composition over almost the whole length of the ingot can be achieved. Secondly, the growth temperature used is relatively low. However, the concentration of the species in the solution is significantly lower than in a comparable melt. Therefore, the material transport through the solution zone is the limiting step and only extremely low growth rates have been used to date (1-5 mm day $\left.{ }^{-1}\right)$ [1-4].

The accelerated crucible rotation technique (ACRT) has been well studied by Schulz-DuBois who gave a detailed description of the convective flows produccd by ACRT [5]. It is one tool for effectively stirring the solution and consequently, to raise the supply of particles to the growing interface. In this way ACRT is considered to allow a higher growth rate. This technique was first used by Scheel [6] to grow crystals of garnets from high-temperature solutions. Further studies have been on the application of ACRT to THM growth of CdTe crystals [7] and to Bridgman growth of $\mathrm{Hg}_{1-x} \mathrm{Cd}_{x} \mathrm{Te}$ alloys [8-12].

Whereas there has been a patent on this subject [13], this is the first report in the open literature on the THM crystal growth of $\mathrm{Hg}_{1-x} \mathrm{Cd}_{x} \mathrm{Te}$ using ACRT. During the experiments, the effect of a significant increase in growth rate on the perfection of the growing crystals was studied. The aim was to carry out THM crystal growth with ACRT at higher growth rates but without degrading the structural perfection.

\section{Theory}

Referring to the growth mechanism of THM, only a simple one-dimensional model necd be considered. The growth rate is determined by the translation of the furnace as long as the material transport through the solution zone allows the growing interface to advance. The maximum rate limit of a macroscopically smooth interface is given by the concept of the constitutional supercooling criterion. An analytical description of this maximum rate has been established by Tiller et 
al. [14]. In the case of crystal growth by THM this criterion can be written as:

$v_{\mathrm{cs}}=\frac{D c_{\mathrm{L}} \Delta H_{\mathrm{s}} G_{\mathrm{L}}}{\left(c_{\mathrm{sg}}-c_{\mathrm{lg}}\right) R T_{\mathrm{L}}^{2}}$,

where $v_{\mathrm{cs}}$ is the maximum stable growth rate, $\Delta H_{\mathrm{s}}$ the latent heat of crystallization, $G_{\mathrm{L}}$ the temperature gradient in the liquid phase next to the interface given by the furnace, $R$ the gas constant, $T_{\mathrm{L}}$ the absolute temperature at the solid-liquid interface, $D$ the diffusion coefficient, $c_{\mathrm{lg}}$ and $c_{\mathrm{sg}}$ are the concentrations of the crystallizing species in the liquid and the solid phases in front of the interface, and $c_{\mathrm{L}}$ is the average value of the concentration of the crystallizing species in the liquid phase.

This means that a macroscopically smooth interface changes to a cellular substructure if the actual temperature at some point in front of the interface becomes lower than the equilibrium temperature of the actual concentration of the species in the solvent at just this position. Other authors dealing with crystal growth with ACRT [6-12] have not given any basic explanation for the reasons why forced convection can increase the maximum growth rate limit. This will be attempted in the following paragraph.

Convectional stirring causes a relatively homogeneous composition in the interior part of the zone, whereas concentration gradients are formed in the regions adjacent to the interfaces. The gradient $\partial c / \partial z$ causes a diffusion flux $j_{\mathrm{D}}$ through the interface which is given by the equation

$j_{\mathrm{D}}=-D \frac{\partial c}{\partial z}=-\frac{D}{m} \frac{T_{\mathrm{d}}-T_{\mathrm{g}}}{2 \delta}$

where $\delta$ is the thickness of the region in which the concentration gradient exists, $T_{\mathrm{d}}$ and $T_{\mathrm{g}}$ are the temperatures at the dissolving and the growing interfaces, and $m=\partial c / \partial T$ is the slope of the liquidus in the phase diagram of the crystal and the solvent. A schematic representation of the concentration profile within the liquid zone is given in fig. 1. With the conservation of mass equation at the growing interface

$v\left(c_{\mathrm{sg}}-c_{\mathrm{lg}}\right)=\left.D \frac{\partial c}{\partial z}\right|_{z=0}$

(where $v$ is the growth rate) and a fixed temperature, the growth rate is always proportional to the

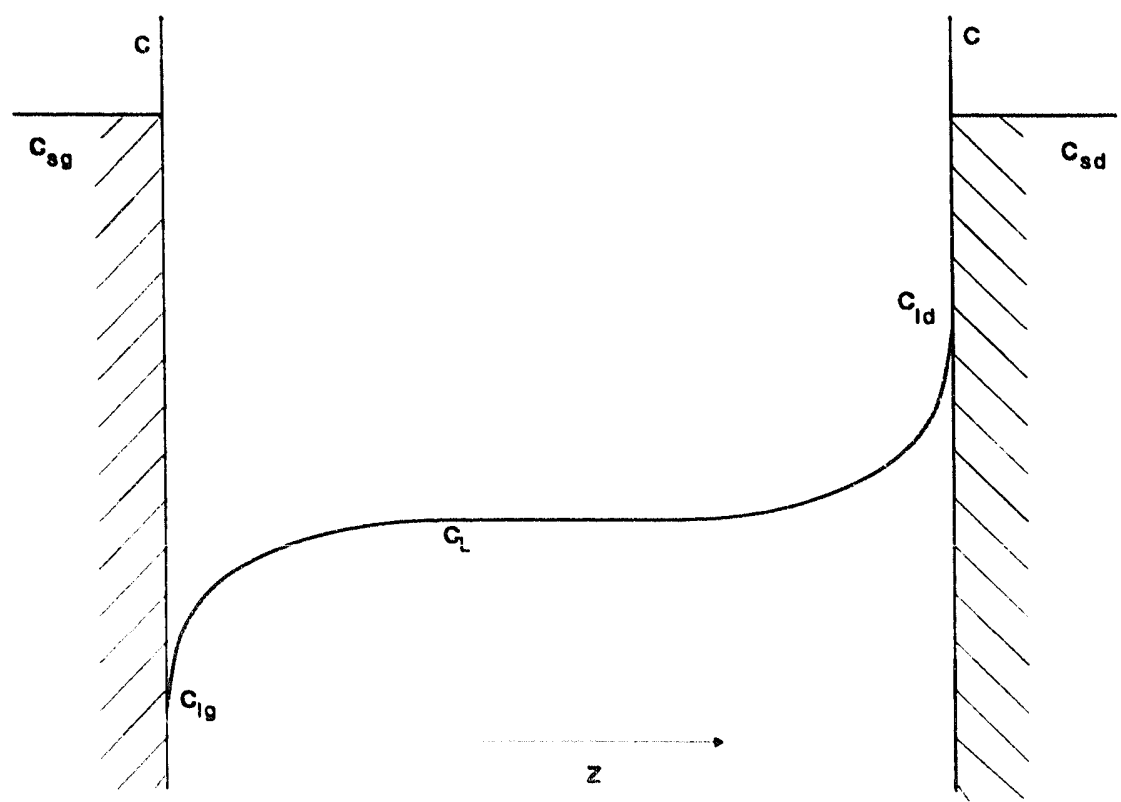

Fig. 1 . Schematic representation of the concentration profile of a component in the liquid zone (I, liquid; $s$, solid; $L$, average value in the liquid; g, growing; $d$. dissolving). 
concentration gradient at the interface. Thus, the concentration gradient adjacent to the interface is independent of the stirring regime or other parameters according to the mass transport conditions, as long as the growth rate is held constant. ACRT, too, cannot vary the proportionality between growth rate and concentration gradient, and the fundamental statement of the constitutional supercooling criterion, e.g. in terms of eq. (1), stays unchanged. The question arises of how the effectiveness of any technique of stirring may be understood by simple constitutional supercooling arguments? What is influenced, e.g., by ACRT is the extension of concentration gradients. With convective stirring, the profile of fluid motion and the resultant profile of concentration are already changed close to the growing interface, although the gradient next to the interface stays constant. The change in the absolute value of the concentration of the species at the interface caused by ACRT is small. For both, forced and ordinary convection, therefore, the extension and the absolute quantity of constitutional supercooling are different (see fig. 2).

As crystal growth is a process of statistics of growth and dissolution, and the simple criterion of eq. (1) is not always strictly fulfilled, the much smaller extension of a supercooled region in coordinates of distance and temperature explains the question of relative stability. It follows, therefore, that ACRT, as well as other techniques of forced convection, drastically change the limits of growth rate. If the growth rate is so high that unstable growth occurs, parts of the solution are incorporated into the crystal as mother liquid inclusions. For this reason, the density and the size of such inclusions in a grown crystal can be a characteristic value to determine whether the maximal growth rate limit is exceeded.

In the THM type of geometry the Ekman layer flow exerts the main influence on mixing the solution during growth. The pattern of this flow is shown in ref. [5]. Equations determining the order of magnitude of the characteristic values are given by Schulz-DuBois [5]. The Ekman layer flow persists only during the transient spinup/spin-down interval until the liquid achieves the state of uniform rotation. This time is of the order of:

$t=1 / \sqrt{E} \Omega_{0}=R \sqrt{\rho / \eta \Omega_{0}}$,

with

$E=\eta / \rho \Omega_{0} R^{2}$,

where $E$ is the Ekman number, $\eta$ the dynamic viscosity, $\rho$ the density, $\Omega_{0}$ the maximum rotation rate, and $R$ the characteristic length (e.g. the radius of the ampoule).
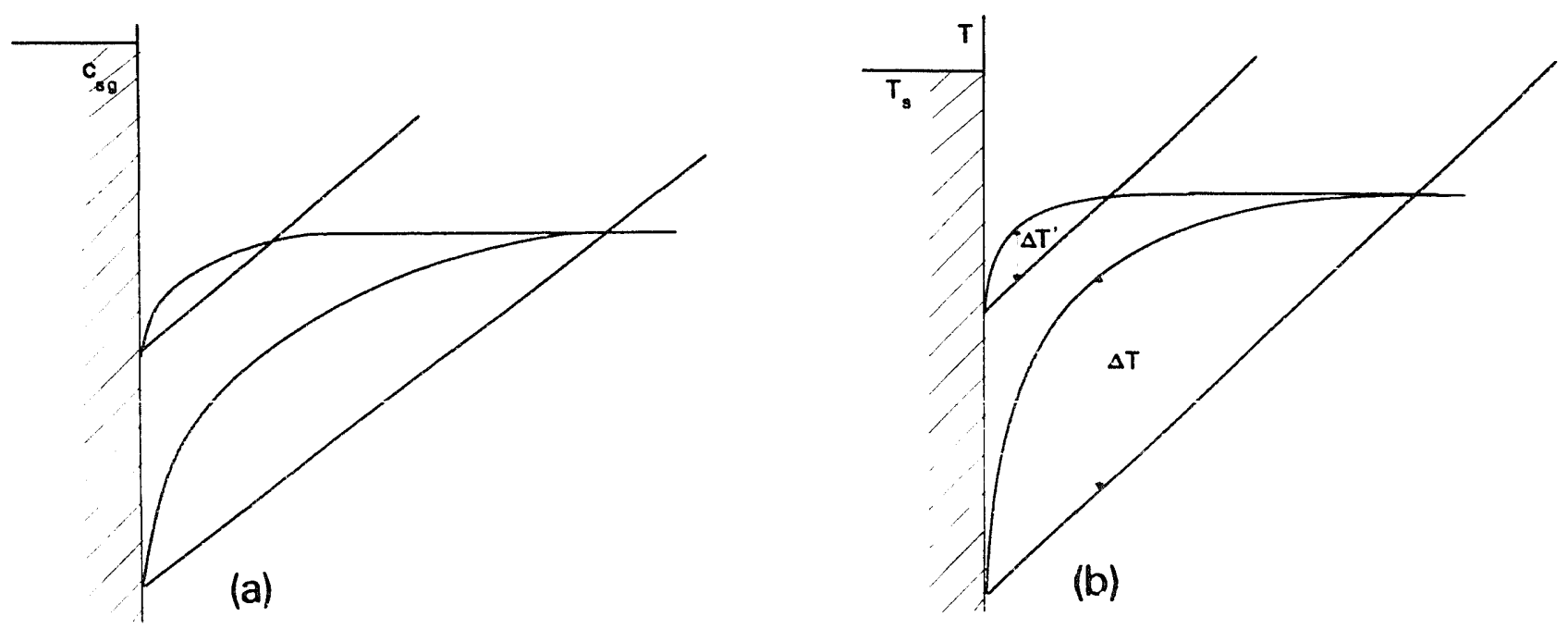

Fig. 2. (a) Concentration profiles in front of the growing interface without and with forced convection and (b) corresponding equilibrium temperature profiles in both cases (the length of the arrows characterizes the amount of supercooling). 
The rapid radial flow in the Ekman layer, which has a thickness of the order of

$d=R \sqrt{E}=\sqrt{\eta / \rho \Omega_{0}}$,

can expand the region of convectional mixing in the direction of both the growing and the dissolving interfaces.

\section{Experimental procedure}

In the application of the ACRT to growth of $\mathrm{Hg}_{1-x} \mathrm{Cd}_{x} \mathrm{Te}$ single crystals there are two questions which can be answered independently. First, the optimum ACRT cycle must be selected from all possible ones and second, the maximum growth rate limit with this cycle has to be determined. The optimum ACRT cycle should be defined as a compromise on high acceleration rates enhancing fluid flow and growth conditions being as continuous as to prevent the crystal from recording the oscillatory nature of ACRT. The spin-up and spin-down times were calculated using eq. (4) and the relevant geometric and hydrodynamic parameters for the $\mathrm{Hg}_{1-x} \mathrm{Cd}_{x} \mathrm{Te}$ growth experiments $\left(\eta=\eta_{\text {tellurium }}=0.00915 \mathrm{~g} \mathrm{~cm}^{-1} \mathrm{~s}^{-1}\right.$ [7], $\rho=7.47 \mathrm{~g}$ $\left.\mathrm{cm}^{-3}, R=8 \mathrm{~mm}, \Omega_{0}=200 \mathrm{rpm}\right)$. Apart from unstable Ekman flow resulting from extreme ac-

a)

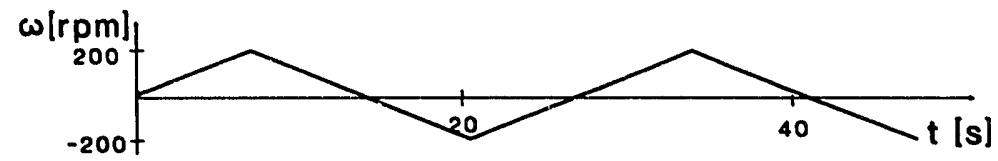

b)

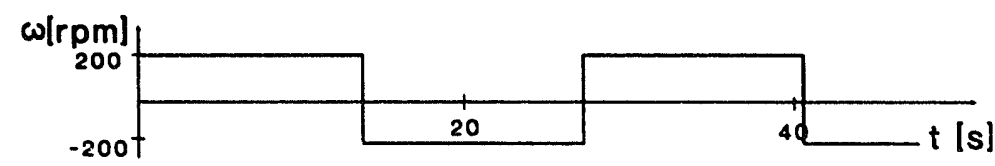

c)

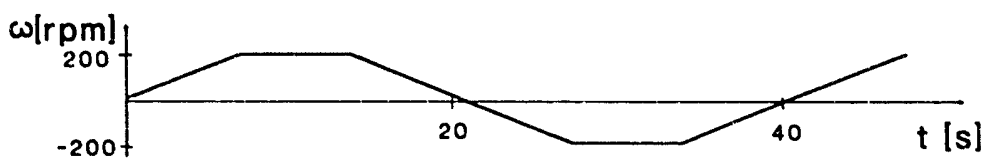

d)

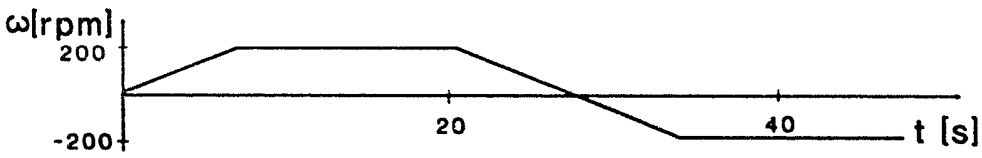

e)

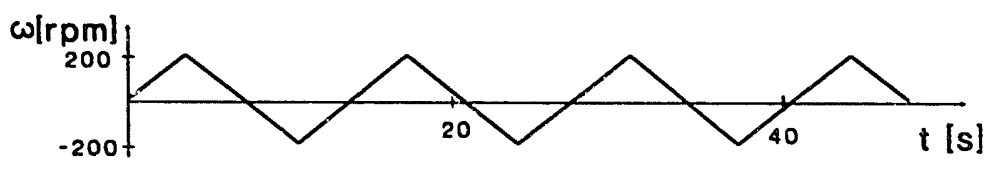

)

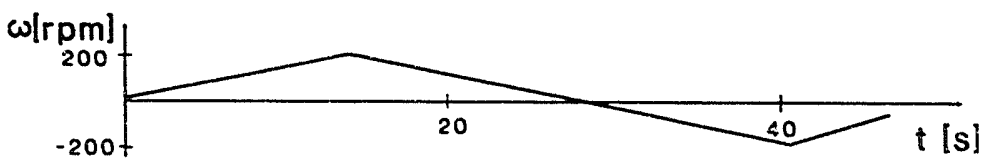

g)

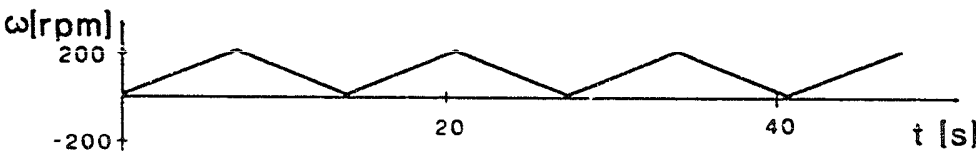

h)

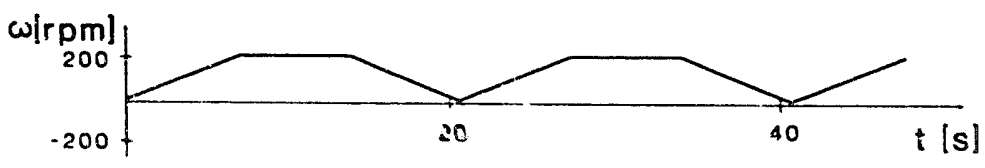

Fig. 3. ACRT cycles used during this study. 
celerations that should be avoided, the maximum rotation rate $\Omega_{0}$ was given by the mechanical stability of the growth equipment. The cycle following from this is shown in fig. 3a. Some variations were carried out to investigate their influence on the growing crystals:

(i) the period and the maximum rotation rate were held constant, but instead of accelerated or decelerated phases, only those of uniform rotation rate were used (fig. 3b);

(ii) the amounts of acceleration and deceleration were held constant, but phases of uniform rotation were added (figs. $3 \mathrm{c}$ and $3 \mathrm{~d}$ );

(iii) the phases were shortened or extended (figs. $3 \mathrm{e}$ and $3 \mathrm{f}$ );

(iv) the reversion of rotation was omitted (figs. $3 \mathrm{~g}$ and $3 h$ ).

It was desirable to select that cycle which satisfies the conditions for enhancing Ekman layer flow. On the other hand, too strong a fluctuation in growth conditions should be avoided. All crystals were grown under vacuum $\left(10^{-4} \mathrm{~Pa}\right)$ in closed silica crucibles which had been coated with graphite. Use was made of $\langle 111\rangle$ or $\langle\overline{1} \overline{1}\rangle$ oriented CdTe seeds, unsaturated tellurium zones and $\mathrm{Hg}_{1-x} \mathrm{Cd}_{x} \mathrm{Te}$ feeds $(x=0.22)$. The growth temperature was chosen as $500 \pm 10^{\circ} \mathrm{C}$.

The crystals were investigated by electron probe microanalysis (EPMA) using $C d L \alpha$ radiation in order to study their metallurgical homogeneity and by optical microscopy with respect to the density and the distribution of the solvent inclusions. The results were related to those conditions having influenced the growth of the crystal segment under investigation.

\section{Results and conclusion}

All $\mathrm{Hg}_{1-x} \mathrm{Cd}_{x} \mathrm{Te}$ crystals grown during this study were monocrystalline. This result was independent of the ACRT cycle. The structural perfection did not show a significant degradation with increasing growth rate from 1.5 up to 8.5 $\mathrm{mm} \mathrm{day}^{-1}$. The investigation of the axially macroscopic distribution has shown that ACRT has no

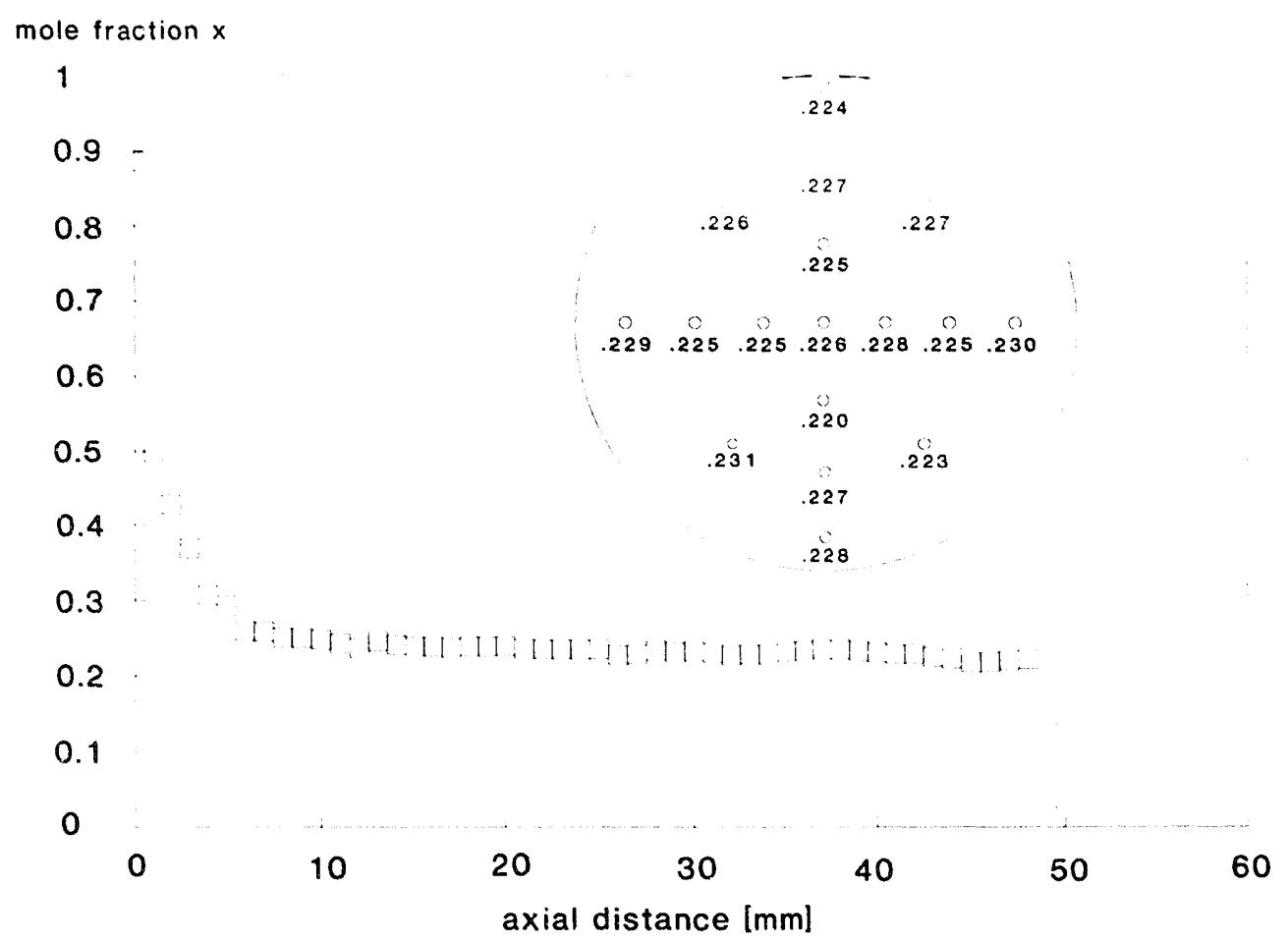

Fig. 4. Electron probe microanalysis measurements of the CdTe mole fraction $x$ in a grown $\mathrm{Hg}_{1-x} \mathrm{Cd}_{x}$ Te crystal along its axis and on a $16 \mathrm{~mm}$ diameter slice cut from the crystal perpendicular to this axis. 
influence on the metallurgical homogeneity of THM-grown $\mathrm{Hg}_{1-x} \mathrm{Cd}_{x} \mathrm{Te}$ crystals. A typical axial distribution of the mole fraction is represented in fig. 4. The whole region following the CdTe seed and the transient region has the composition which is equal to that of the feed ingot.

In principle, because of the oscillatory nature of ACRT, the risk of a compositional fluctuation in a microscopic scale (striations) exists. With the ACRT periods and translation rates used, these striations should have a spacing in the range between 0.35 and $2.0 \mu \mathrm{m}$, but such striations would not be detected by EPMA using 2-3 $\mu \mathrm{m}$ beam diameter. However, calculations by Gray et al. [15] have suggested, that hypothetic striations in $\mathrm{Hg}_{1-x} \mathrm{Cd}_{x} \mathrm{Te}$ would rapidly decay by solid-state diffusion because of the high self-diffusion coefficient $\left(D=4.41 \times 10^{-11} \mathrm{~cm}^{2} \mathrm{~s}^{-1}\right.$ at $500^{\circ} \mathrm{C}, x=$ 0.21 [16]). This is in agreement with the ACRT Bridgman growth experiments [10,12], which have used a growth rate of $0.5 \mathrm{~mm} \mathrm{~h}^{-1}$ and an ACRT sequence of spin-up for $8 \mathrm{~s}$ and spin-down of $1 \mathrm{~s}$. The authors did not observe striations. In our experiments, if striations occur, the spacing from ACRT would lie in the same range, and should not be stable.

Furthermore, it is feasible that high rotation rates cause a separation between the heavier and the lighter components because of the different centrifugal forces. This can be disproved by measurements of the radial mole fraction distribution on slices cut perpendicular to the growth axis (fig. 4). The differences in the mole fraction between the individual points are within the experimental error of the measurement and show no separation tendency. This is in good agreement with simple estimations on centrifugal forces for the small diameter and moderate rotation rates used. Both, axial and radial $x$ distribution, do not differ from the results we obtained in a non-ACRT regime [4].

As mentioned above, generally, there is a high risk of mother liquid inclusion in solution grown crystals. Therefore, a rough value limiting the growth rate is achieved if the density of tellurium inclusions becomes higher. To date an cxact investigation of the dependence of the inclusion density on the growth rates in THM grown
$\mathrm{Hg}_{1-x} \mathrm{Cd}_{x} \mathrm{Te}$ has not been published. It can be assumed, however, that the extremely low growth rates in the usual experiments [1-4] have been used because of the high risk of mother liquid inclusions. With ACRT applied to THM, no increase in tellurium inclusion density was found up to growth rates of $8.5 \mathrm{~mm}$ day $^{-1}$. Any dependence of the density and the distribution of inclusions on the different ACRT cycles applied could not be revealed with the growth rates used in this study.

However, it should be noted that only those inclusions intersecting the surfaces of the slices can be detected by optical microscopy, since $\mathrm{Hg}_{1-x} \mathrm{Cd}_{x} \mathrm{Te}$ is opaque in the range of visible and infrared light up to several $\mu \mathrm{m}$. Therefore, further basic experiments will be carried out with material, e.g. CdTe, ZnTe or $(\mathrm{Cd}, \mathrm{Zn}) \mathrm{Te}$, being transparent for the light used for the investigation. Nevertheless, $\mathrm{Hg}_{1-x} \mathrm{Cd}_{x} \mathrm{Te}$ and its $\mathrm{Hg}$-based substitutes will be the preferred substances for the practical use of ACRT-assisted THM.

\section{Summary}

The growth of $\mathrm{Hg}_{1-x} \mathrm{Cd}_{x} \mathrm{Te}$ single crystals by THM using ACRT was preved to be possible. By extending the region of convective mixing towards the interfaces, the growth rate can be increased, as predicted by theoretical considerations. An effective way of stirring the solution zone in front of the interfaces was achieved by the use of cycles which satisfy the conditions to enhance the Ekman flow. The density of tellurium inclusions did not increase markedly with increasing growth rates up to $8.5 \mathrm{~mm} \mathrm{day}^{-1}$. The microscopic and macroscopic homogeneity were not degraded relative to crystals grown without ACRT at much lower rates.

\section{Acknowledgments}

The authors are grateful to Dr. N. Puhlmann for carrying out the electron probe microanalysis measurements. This work was supported by the Volkswagen-Stiftung under contract No. I/65988. 


\section{References}

[1] R. Triboulet, T. Nguyen Duy and A. Durand, J. Vacuum Sci. Technol. A 3 (1985) 95.

[2] B. Baird, P.-K. Liao, R. Chang and L. Colombo, J. Crystal Growth 98 (1989) 595.

[3] I. Shilo, E. Kedar and D. Szafranek, Mater. Res. Soc. Symp. Proc. 214 (1990) 47.

[4] P. Gille, F.M. Kiessling and M. Burkert, J. Crystal Growth 114 (1991) 77.

[5] E.O. Schulz-DuBois, J. Crystal Growth 12 (1972) 81.

[6] H.J. Scheel, J. Crystal Growth 13/14 (1972) 560.

[7] F.V. Wald and R.O. Bell, J. Crystal Growth 30 (1975) 29.

[8] P. Capper, W.G. Coates, C.L. Jones, J.J. Gosney, C.K. Ard and I. Kenworthy, J. Crystal Growth 83 (1987) 69.
[9] W.G. Coates, P. Capper, C.L. Jones, J.J. Gosney, C.K. Ard and I. Kenworthy, J. Crystal Growth 94 (1989) 559.

[10] P. Capper, J.J. Gosney and C.L. Jones, J. Crystal Growth 70 (1984) 356.

[11] P. Capper, J.J. Gosney, C.L. Jones and E.J. Pearce, J. Electron. Mater. 15 (1986) 361.

[12] P. Capper, J.J. Gosney, C.L. Jones and I. Kenworthy, J. Electron. Mater. 15 (1986) 371.

[13] Patent No. 8804370 (1988), Procédé de préparation d'un lingot cristallin de $\mathrm{HgCdTe}$, by $\mathrm{M}$. Royer, B.R. Jean. A.R. Durand and R.G. Triboulet.

[14] W.A. Tiller, K.A. Jackson, J.W. Rutter and B. Chalmers, Acta Met. 1 (1953) 428.

[15] R.T. Gray, M.F. Larrousse and W.R. Wilcox, J. Crystal Growth 92 (1988) 530.

[16] D. Shaw, Phil. Mag. A 53 (1986) 727, quoted in ref. [15]. 